BMJ Open Diabetes Research \& Care

\section{Undiagnosed diabetes and impaired fasting glucose in HFE C282Y homozygotes and HFE wild-type controls in the HEIRS Study}

To cite: Barton JC, Barton JC, Adams PC, et al. Undiagnosed diabetes and impaired fasting glucose in HFE C282Y homozygotes and HFE wild-type controls in the HEIRS Study. BMJ Open Diabetes Research and Care 2016:4:e000278. doi:10.1136/bmjdrc-2016000278

- Additional material is available. To view please visit the journal (http://dx.doi.org/ 10.1136/bmjdrc-2016000278)

Received 29 May 2016 Revised 4 November 2016 Accepted 14 November 2016

\section{(a) CrossMark}

${ }^{1}$ Southern Iron Disorders Center, Birmingham, Alabama, USA

${ }^{2}$ Department of Medicine, University of Alabama at Birmingham, Birmingham, Alabama, USA

${ }^{3}$ Department of Medicine, University of Western Ontario, London, Ontario, Canada

${ }^{4}$ Department of Microbiology, University of Alabama at Birmingham, Birmingham, Alabama, USA

Correspondence to Dr James C Barton; ironmd@isp.com

\author{
James C Barton, ${ }^{1,2} \mathrm{~J}$ Clayborn Barton, ${ }^{1}$ Paul C Adams, ${ }^{3}$ Ronald T Acton ${ }^{1,4}$
}

\section{ABSTRACT}

Objective: To determine prevalences and predictors of undiagnosed diabetes mellitus (UDM) and impaired fasting glucose (IFG) in non-Hispanic whites with HFE p.C282Y homozygosity and controls without common HFE mutations identified in population screening.

Research design and methods: We analyzed these observations in a postscreening examination: age; sex; body mass index; systolic/diastolic blood pressure; metacarpophalangeal joint hypertrophy; hepatomegaly; blood neutrophils; alanine and aspartate aminotransferase; elevated $\mathrm{C}$ reactive protein; transferrin saturation; serum ferritin; and Field Center. Results: There were 223 p.C282Y homozygotes and 449 controls without diagnosed diabetes $(43.9 \%$ men). Mean age of $p . C 282 Y$ homozygotes was $52 \pm 13$ years (controls $57 \pm 14$ years; $p<0.0001$ ). Mean transferrin saturation in p.C282Y homozygotes was $67 \pm 26 \%$ (controls $34 \pm 14 \% ; p<0.0001$ ). Mean serum ferritin in p.C282Y homozygotes was $607 \mathrm{pmol} / \mathrm{L}(95 \% \mathrm{Cl} 497$ to 517 ; controls $274 \mathrm{pmol} / \mathrm{L}$ (247 to 301$)$; $p<0.0001$ ). Overall prevalences of UDM (4.0\% vs $4.2 \%)$ and IFG $(23.8 \%$ vs $25.6 \%)$ did not differ significantly between p.C282Y homozygotes and wt/wt controls, respectively. In logistic regressions, male sex, body mass index, and alanine aminotransferase were significantly associated with UDM. ORs were 2.7 (1.2 to 2.8); 1.0 (1.0 to 1.1 ); and 1.0 (1.0 to 1.0$)$, respectively. Age, male sex, and body mass index were significantly associated with IFG. ORs were 1.0 (1.0 to 1.1 ); 2.8 (1.9 to 4.2); and 1.0 (1.0 to 1.1), respectively.

Conclusions: Prevalences of UDM and IFG were similar in p.C282Y homozygotes and controls in a postpopulation screening examination. Male sex was the strongest predictor of UDM and IFG.

HFE hemochromatosis is an autosomal recessive condition ${ }^{1}{ }^{2}$ due to homozygosity for the p.C282Y mutation of the HFE gene on chromosome 6p21.3. ${ }^{3}$ HFE hemochromatosis occurs in $0.3-0.6 \%$ of persons of European descent. ${ }^{3-5}$ p.C282Y homozygosity accounts for $\sim 90 \%$ of 'classical' hemochromatosis iron phenotypes in whites. ${ }^{3}{ }^{4}$ Iron overload in p.C282Y homozygotes, especially if severe, may cause diabetes mellitus,

\section{Significance of this study}

What is already known about this subject?

- There are no previous reports of the prevalence of undiagnosed diabetes mellitus and impaired fasting glucose in HFE p.C282Y homozygotes identified by screening.

What are the new findings?

- The respective prevalences of undiagnosed diabetes mellitus and impaired fasting glucose were similar in non-Hispanic white p.C282Y homozygotes and matched controls without common HFE mutations in this cross-sectional, postpopulation screening examination. In multivariable analyses, male sex was the strongest independent variable associated with both undiagnosed diabetes mellitus and impaired fasting glucose. Serum ferritin level was not significantly associated either with undiagnosed diabetes mellitus or impaired fasting glucose.

How might these results change the focus of research or clinical practice?

- Factors other than serum ferritin or iron overload contribute to undiagnosed diabetes mellitus and impaired fasting glucose risks in p.C282Y homozygotes. Longitudinal studies of other cohorts may provide information about the incidence of undiagnosed diabetes mellitus and impaired fasting glucose in p.C282Y homozygotes.

other endocrinopathies, cirrhosis, primary liver cancer, and cardiomyopathy. ${ }^{6}$

Diabetes was diagnosed in $\sim 80 \%$ of patients with hemochromatosis reported from the late 19th century to the mid-20th century. ${ }^{7}$ Most patients with hemochromatosis and diabetes also had heavy liver iron loading and cirrhosis. ${ }^{7}$ Early investigators attributed diabetes in persons with hemochromatosis to ironinduced fibrosis of the pancreatic acini and islets of Langerhans ${ }^{78}$ and specificity of iron deposition for the $\beta$ cells of the islets. ${ }^{9}{ }^{10}$ The results of population screening studies of the 21st century revealed that the prevalence of 
type 2 diabetes in p.C282Y homozygotes and persons without common HFE mutations is similar. ${ }^{11-14}$ Today, increased type 2 diabetes risk in persons with HFE hemochromatosis is associated with one or more factors, including severe iron overload, ${ }^{78}{ }^{15}$ decreased insulin secretion, ${ }^{15}{ }^{16}$ cirrhosis, ${ }^{6}$ history of diabetes in firstdegree relatives, ${ }^{17-19}$ increased body mass index, ${ }^{19-22}$ insulin resistance, ${ }^{21}$ and metabolic syndrome. ${ }^{22}$

In the US National Health and Nutrition Examination Survey (NHANES) 1999-2002, approximately one-third of participants with diabetes had previously undiagnosed diabetes mellitus (UDM). ${ }^{23}$ Impaired fasting glucose (IFG) occurred in more than $25 \%$ of non-Hispanic whites in NHANES 1999-2002. ${ }^{23}$ In a previous report, we identified risk factors for diagnosed diabetes in p.C282Y homozygotes who participated in a postscreening clinical examination of the Hemochromatosis and Iron Overload Screening (HEIRS) Study. ${ }^{22}$ We also postulated that the respective prevalences of UDM and IFG in p.C282Y homozygotes and control subjects who lacked common missense in HFE (wt/wt) do not differ significantly. To learn more about UDM and IFG in p.C282Y homozygotes, we evaluated observations from non-Hispanic white HEIRS Study participants without diagnosed diabetes who participated in a postscreening examination. We determined prevalences and independent predictors of UDM and IFG in HFE p.C282Y homozygotes and control participants without common HFE mutations. We compared our observations with those of UDM and IFG in populations of non-Hispanic whites in NHANES 1999-2002 23 and discuss the implications of our results for understanding diabetes risk in persons with HFE hemochromatosis.

\section{RESEARCH DESIGN AND METHODS}

Participants and initial screening

The National Heart, Lung, and Blood Institute/National Human Genome Research Institute HEIRS Study investigators evaluated the prevalence, genetic, and environmental determinants, and potential clinical, personal, and societal impacts of hemochromatosis and iron overload in a multiethnic, primary care-based, cross-sectional sample of 101168 adults enrolled during the interval 2001-2003 at four Field Centers in the USA and one in Canada. ${ }^{24}$ The Study was conducted in accordance with the principles of the Declaration of Helsinki. Participants $\geq 25$ years of age and able to give informed consent were recruited from a health maintenance organization, diagnostic blood collection centers, and public and private primary care offices in ambulatory clinics associated with the Field Centers. ${ }^{24}$ Initial screening of participants, performed in 2000-2002, included iron phenotyping and genotyping for HFE p.C282Y and p.H63D alleles. ${ }^{24}$

\section{Clinical examination}

In accordance with the HEIRS Study design, ${ }^{14}$ clinical examination participants designated as cases included all p.C282Y homozygotes and other participants with high-iron phenotypes, regardless of HFE genotype. ${ }^{14}$ Before the clinical examination, control subjects in three broad age groups (25 to $45,>45$ to 65 , and $>65$ years) were selected in a 1: 1 ratio with all p.C282Y homozygotes identified at each of the five Field Centers. Control subjects were selected from participants with $H F E \mathrm{wt} / \mathrm{wt}$, without elevations in transferrin saturation (TS) and serum ferritin (SF), and with TS and SF within the middle half of the respective gender-specific distributions. $^{24}$ The present study evaluated postscreening clinical examination observations in non-Hispanic white p.C282Y homozygotes and wt/wt control participants.

The median interval between initial screening and clinical examination participation was 8 months. The clinical examination included a questionnaire addressing medical history and medications that was completed by the participant and a focused physical examination performed by a HEIRS Study physician. ${ }^{14}$ Body mass index was computed as $\mathrm{kg} / \mathrm{m}^{2}$. Systolic and diastolic blood pressures were measured using the auscultatory method and a mercury or aneroid sphygmomanometer. Participants were evaluated for hypertrophy of the second and third metacarpophalangeal joints, the most distinctive characteristic of hemochromatosis arthropathy. ${ }^{6} 24$ Obtaining accounts of previous diagnoses of hemochromatosis or phlebotomy therapy was beyond the scope of the HEIRS Study.

At clinical examination, a morning blood sample was obtained after an overnight fast of $\geq 8$ hours for: confirmatory HFE genotyping; ${ }^{14}$ complete blood counts (Beckman Coulter GenS, Beckman/Coulter, Fullerton, California, USA); serum alanine and aspartate aminotransferase activities; serum $\mathrm{C}$ reactive protein; TS and SF (Hitachi 9/11 Analyzer, Roche Applied Science, Indianapolis, Indiana, USA); and serum glucose (Hitachi 9/11 Analyzer, Roche Applied Science, Madison, Wisconsin, USA). ${ }^{14}{ }^{25}$ Using control specimens that represented normal ranges of SF, the total coefficient of variation for the Hitachi 9/11 Analyzer was $5.82-6.78 \%$. For higher range SF standards, the total coefficient of variation was $5.98-8.24 \% .^{26}$ All testing was performed at the HEIRS Study Central Laboratory (Fairview-University Medical Center Clinical Laboratory, University of Minnesota, Fairview, Minnesota, USA). In participants with elevated alanine aminotransferase, reflex testing for hepatitis $\mathrm{B}$ surface antigen and hepatitis C antibody was performed (Vitros ECi, Ortho-Clinical Diagnostics Incorporated). Reference ranges for these analytes are displayed in the footnotes of table 1 .

\section{Participant exclusions}

The data set included observations on 1129 non-Hispanic whites, among whom were 285 p.C282Y homozygotes and $523 \mathrm{wt} / \mathrm{wt}$ control participants. We excluded 62 p.C282Y homozygotes (22 diagnosed diabetes or previously diagnosed hemochromatosis; 10 
Table 1 Characteristics of 672 HEIRS Study participants without diagnosed diabetes*

\begin{tabular}{|c|c|c|c|}
\hline Characteristic & $\begin{array}{l}\text { HFE p.C282Y } \\
\text { homozygotes }(n=223)\end{array}$ & $\begin{array}{l}\text { HFE wt homozygotes } \\
(\mathrm{n}=449)\end{array}$ & Value of pt \\
\hline Mean age, years & 30I & & $<0.0001$ \\
\hline Male, percent (n) & $40.3(91)$ & $45.4(204)$ & \\
\hline Mean body mass index, $\mathrm{kg} / \mathrm{m}^{2}$ & $28.2 \pm 5.7$ & $28.3 \pm 5.8$ & 0.8831 \\
\hline Mean systolic blood pressure, $\mathrm{mm} \mathrm{Hg}$ & $125 \pm 17$ & $124 \pm 17$ & \\
\hline Mean diastolic blood pressure, $\mathrm{mm} \mathrm{Hg}$ & $78 \pm 11$ & $76 \pm 10$ & 0.0 \\
\hline Hepatomegaly, percent (n) & 0) & 4) & 5 \\
\hline Metacarpophalangeal joint hypertrophy, percent (n) & $11.7(26)$ & $6.0(27)$ & 0. \\
\hline Mean blood neutrophils $\times 10^{9} / \mathrm{L}$ & $3.8 \pm 1.7$ & $3.6 \pm 1.6$ & 44 \\
\hline Mean alanine aminotransferase, $\mu \mathrm{kat} / \mathrm{L}$ & 8 & .48 & \\
\hline Mean aspartate aminotransferase, $\mu \mathrm{kat} / \mathrm{L}$ & $0.42 \pm 0.32$ & $0.48 \pm 0.47$ & 0.0328 \\
\hline Elevated C reactive protein, percent $(n)$ & $32.7(73)$ & $29.0(130)$ & 272 \\
\hline Mean transferrin saturation, percent & $67 \pm 26$ & $34 \pm$ & $<0.0$ \\
\hline Mean serum ferritin, $\mathrm{pmol} / \mathrm{L}$ & 607 (497 to 517 ) & 274 (247 to 301$)$ & $<0.0001$ \\
\hline Undiagnosed diabetes, percent $(95 \% \mathrm{Cl})(\mathrm{n}) \ddagger$ & 4.0 (9) (2.0 to 7.8$)$ & 4.2 (19) (2.6 to 6.7$)$ & 00 \\
\hline Impaired fasting glucose, percent $(95 \% \mathrm{Cl})(\mathrm{n}) \ddagger$ & $23.8(53)(18.5$ to 30.0$)$ & $25.6(115)(21.7$ to 30.0$)$ & 0.6369 \\
\hline \multicolumn{4}{|c|}{ 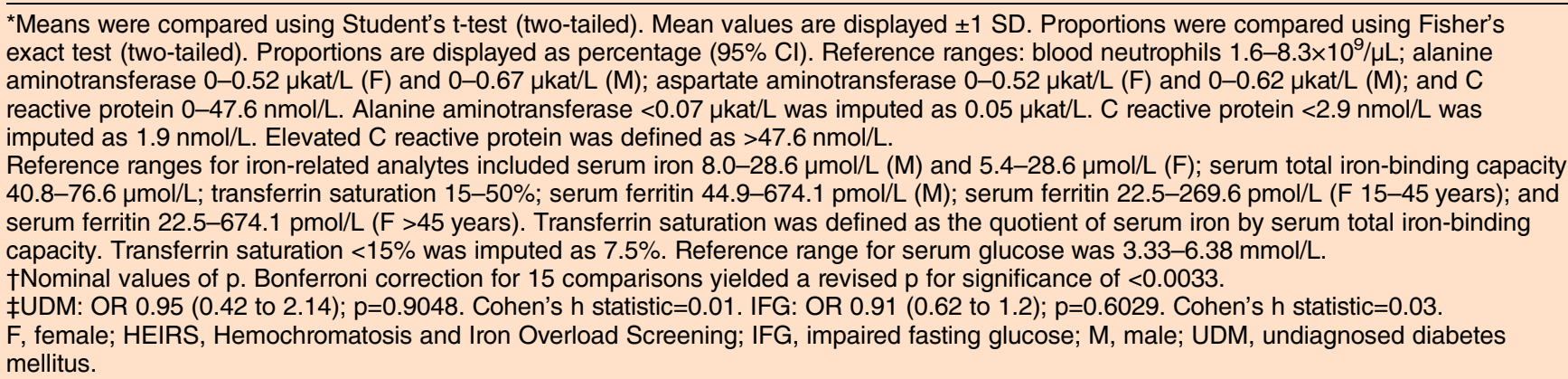 } \\
\hline
\end{tabular}

pregnancy; 30 missing data, overnight fast $<8$ hours, report of cirrhosis, or positivity for hepatitis B or C) and 74 control participants (27 diagnosed diabetes; 8 pregnancy; 39 missing data, overnight fast $<8$ hours, report of cirrhosis, or positivity for hepatitis B or C). We excluded participants with reports of cirrhosis because we could not substantiate these reports. Individual prevalences of diagnosed diabetes, pregnancy, missing data, overnight fast $<8$ hours, report of cirrhosis, or hepatitis B or C positivity did not differ significantly between p.C282Y homozygotes and wt/wt control participants (data not shown).

\section{Definitions of diagnosed diabetes, UDM, and IFG}

Diabetes diagnoses in participants classified by selfreports at initial screening were confirmed at clinical examination by questionnaire and reviews of medications and medication lists. We defined UDM as fasting serum glucose $\geq 6.99 \mathrm{mmol} / \mathrm{L}$ in participants without diabetes diagnoses. ${ }^{27} 28$ We defined IFG as an elevated fasting serum glucose $\geq 5.55$ and $<6.99 \mathrm{mmol} / \mathrm{L}$ in participants without diabetes diagnoses. ${ }^{27} 28$

\section{Statistics}

The data set consisted of complete observations on 223 p.C282Y homozygotes and 449 wt/wt control participants without diagnosed diabetes from five Field Centers which were pooled for analysis. To summarize diabetes, UDM, and IFG prevalence in HEIRS Study clinical examination participants, previously published observations on 22 p.C282Y homozygotes and $27 \mathrm{wt} / \mathrm{wt}$ controls with diagnosed diabetes ${ }^{22}$ are included in the last of the present tables. Distributions of age, systolic/ diastolic blood pressures, neutrophils, lymphocytes, alanine and aspartate aminotransferase activities, and TS values were normal. We used natural log (ln) transformation to normalize $\mathrm{SF}$ data. Each mean ln-transformed datum was converted to an anti-ln $(95 \%$ CI). Dichotomous variables included HFE genotype; sex; hypertrophy of metacarpophalangeal joints; hepatomegaly; elevated $\mathrm{C}$ reactive protein; and an entry for each of the five Field Centers.

We performed regressions on UDM and IFG in 672 participants using these independent variables: age; sex; body mass index; systolic/diastolic blood pressures; hepatomegaly; metacarpophalangeal joint hypertrophy; blood neutrophils; levels of alanine and aspartate aminotransferases; elevated $\mathrm{C}$ reactive protein; TS; and SF; and Field Center. The models were run unconditional.

Analyses were performed with SAS V.9.1 (SAS Institute, Cary, North Carolina, USA), Excel 2000 (Microsoft Corp, Redmond, Washington, USA), and GB-Stat (V.10.0, Dynamic Microsystems, Silver Spring, Maryland, USA). Descriptive data are displayed as enumerations, proportions $(95 \% \mathrm{CI})$, mean $\pm 1 \mathrm{SD}$, or mean (95\% CI). Means were compared using Student's t-test (two-tailed). Proportions were compared using Fisher's 
exact test (two-tailed). We computed ORs and Cohen's $\mathrm{h}$ statistic as estimates of effect sizes to enhance interpretation of comparisons of proportions involving UDM and IFG prevalence. We performed analyses of covariance to determine whether there were significant interactions of the independent variables age and sex on the respective dependent variables UDM and IFG. ORs (95\% CI) are also displayed for significant variables from logistic regressions. We defined nominal values of $\mathrm{p}<0.05$ to be significant. Bonferroni corrections were applied to control the type I error rate at 0.05 for multiple comparisons of continuous and dichotomous data, as appropriate.

\section{RESULTS}

\section{General characteristics}

Mean age of the 672 participants was $55 \pm 14$ years, 295 $(43.9 \%)$ of whom were men (table 1$)$. There were 223 p.C282Y homozygotes and 449 wt/wt control participants without diagnosed diabetes. p.C282Y homozygotes had lower mean age and higher TS and SF than wt/wt control participants, after Bonferroni correction (table 1).

\section{Age, UDM, and IFG}

The prevalence of UDM was greater in participants aged $\geq 65$ than $<65$ years in p.C282Y homozygotes and wt/wt control participants, but the differences were not significant (table 2). The prevalence of IFG was significantly greater in participants aged $\geq 65$ years in both genotype groups (table 2).

\section{Sex, UDIM, and IFG}

The prevalence of UDM was greater in men than women, although the difference was significant only in wt/wt control participants (table 3). The prevalence of IFG was significantly greater in men than women in both genotype groups (table 3 ).

\section{Interaction of sex and age on UDM and IFG}

Using UDM as the dependent variable and sex and age as the independent variables, the F-value was 5.4210 $(p=0.0200)$ in an analysis of covariance. Using IFG as the dependent variable and sex and age as the independent variables in another analysis of covariance, the F-value was 28.6394 $(\mathrm{p}<0.0001)$. These results suggest that there are significant interactions of age and sex on UDM and IFG.

Table 2 Prevalence of undiagnosed diabetes and impaired fasting glucose by age*

\begin{tabular}{|c|c|c|c|c|c|}
\hline HFE p.C282Y/p.C282Y & Age $<65$ years $(n=184)$ & Age $\geq 65$ years $(n=39)$ & p Value & OR $(95 \% \mathrm{Cl})$ & $\begin{array}{l}\text { Cohen's } \\
\text { h statistic }\end{array}$ \\
\hline $\begin{array}{l}\text { Undiagnosed diabetes, } \\
\text { percent }\end{array}$ & 3.3 (1.3 to 7.3$)(6)$ & 7.7 (2.0 to 22.0 ) (3) & 0.1945 & $\begin{array}{l}0.40(0.10 \text { to } 1.7) \\
p=0.2152\end{array}$ & 0.20 \\
\hline $\begin{array}{l}\text { Impaired fasting glucose, } \\
\text { percent }\end{array}$ & 20.7 (15.2 to 27.4$)(38)$ & 38.5 (23.8 to 55.3) (15) & 0.0230 & $\begin{array}{l}0.42(0.20 \text { to } 0.86) \\
p=0.0199\end{array}$ & 0.87 \\
\hline HFE wt/wt & Age $<65$ years $(n=317)$ & Age $\geq 65$ years $(n=132)$ & p Value & & \\
\hline $\begin{array}{l}\text { Undiagnosed diabetes, } \\
\text { percent }\end{array}$ & 3.5 (1.8 to 6.3$)(11)$ & 6.8 (3.4 to 12.9$)(9)$ & 0.1338 & $\begin{array}{l}0.49(0.20 \text { to } 1.2) \\
p=0.1239\end{array}$ & 0.15 \\
\hline $\begin{array}{l}\text { Impaired fasting glucose, } \\
\text { percent }\end{array}$ & 22.1 (17.7 to 27.1) (70) & 34.1 (26.2 to 42.9$)(45)$ & 0.0092 & $\begin{array}{l}0.56(0.36 \text { to } 0.88) \\
p=0.0115\end{array}$ & 0.27 \\
\hline
\end{tabular}

${ }^{*}$ Estimates based on observations in 672 participants without diagnosed diabetes. Proportions were compared using Fisher's exact test (two-tailed). Proportions are displayed as percentage (95\% confidence limits) (n).

Table 3 Prevalence of undiagnosed diabetes and impaired fasting glucose in men and women*

\begin{tabular}{|c|c|c|c|c|c|}
\hline HFE p.C282Y/p.C282Y & Men $(n=91)$ & Women $(n=132)$ & Value of $p$ & OR $(95 \% \mathrm{Cl})$ & $\begin{array}{l}\text { Cohen's } \\
\text { h statistic }\end{array}$ \\
\hline $\begin{array}{l}\text { Undiagnosed diabetes, } \\
\text { percent }\end{array}$ & 6.6 (2.7 to 14.3$)(6)$ & 2.3 (0.6 to 7.0$)(3)$ & 0.1644 & $\begin{array}{l}3.0(0.7 \text { to } 12.5) ; \\
p=0.1235\end{array}$ & 0.22 \\
\hline $\begin{array}{l}\text { Impaired fasting } \\
\text { glucose, percent }\end{array}$ & 34.1 (24.7 to 44.8 ) (31) & 16.7 (11.0 to 24.4$)(22)$ & 0.0038 & $\begin{array}{l}2.6(1.4 \text { to } 4.8) \\
p=0.0032\end{array}$ & 0.41 \\
\hline HFE wt/wt & Men $(n=204)$ & Women $(n=245)$ & p Value & & \\
\hline $\begin{array}{l}\text { Undiagnosed diabetes, } \\
\text { percent }\end{array}$ & 6.4 (3.6 to 10.9) (13) & $2.9(1.3$ to 6.1$)(7)$ & 0.1059 & $\begin{array}{l}2.3(0.9 \text { to } 5.9) \\
p=0.0797\end{array}$ & 0.21 \\
\hline $\begin{array}{l}\text { Impaired fasting } \\
\text { glucose, percent }\end{array}$ & 35.8 (29.3 to 42.8$)(73)$ & $17.1(15.3,26.8)(42)$ & 0.0001 & $\begin{array}{l}2.7(1.7 \text { to } 4.2) \\
p<0.0001\end{array}$ & 0.43 \\
\hline
\end{tabular}

*Estimates based on observations in 672 participants without diagnosed diabetes. Proportions were compared using Fisher's exact test (two-tailed). Proportions are displayed as percentage (95\% confidence limits) (n). 


\section{Regression on UDM}

In a logistic regression model, there were three significant positive associations: male sex $(\mathrm{p}=0.0220)$; body mass index $(\mathrm{p}=0.0009)$; and alanine aminotransferase $(p=0.0303)$. The OR were 2.7 (1.2 to 2.8$) ; 1.0$ (1.0 to $1.1)$; and 1.0 (1.0 to 1.0$)$, respectively. The $p$ value of this regression was 0.0002 . The regression model accounted for $10.4 \%$ of the deviation of UDM.

\section{Regression on IFG}

In a logistic regression model, there were three significant positive associations: age $(\mathrm{p}=0.0005)$; male sex $(\mathrm{p}<0.0001)$; and body mass index $(\mathrm{p}<0.0001)$. The ORs were 1.0 (1.0 to 1.1 ); 2.8 (1.9 to 4.2 ); and 1.0 (1.0 to $1.1)$, respectively. The $\mathrm{p}$ value of this regression was $\mathrm{p}<0.0001$. The regression model accounted for $10.7 \%$ of the deviation of IFG.

\section{Prevalence of diagnosed diabetes, UDM, and IFG}

The prevalence of diagnosed diabetes was greater in p.C282Y homozygotes than in wt/wt control participants, although this difference was not significant (table 4). Likewise, the prevalence of UDM and IFG did not differ significantly between p.C282Y homozygotes and wt/wt control participants (table 4).

In total, our analyses revealed that UDM accounted for $29.0 \%$ of diabetes in p.C282Y homozygotes and $41.3 \%$ of diabetes in wt/wt control participants $(\mathrm{p}=0.2723)$. Approximately one-quarter of the participants in each genotype group had IFG.

\section{DISCUSSION}

In the present HEIRS Study participants without diagnosed diabetes, the prevalence of UDM as defined by the American Diabetes Association ${ }^{27} 28$ was $3.7 \%$ in p.C282Y homozygotes and $4.0 \%$ in wt/wt controls. The mean age of p.C282Y homozygotes was significantly lower than that of wt/wt control participants. The prevalence of UDM in participants <65 years of age and $\geq 65$ years of age did not differ significantly. Likewise, age was not a significant independent predictor of
UDM. In non-Hispanic white participants in NHANES 1999-2002, the crude prevalence of UDM defined by the same criterion ${ }^{27}$ was $2.9 \%$ and increased from $0.4 \%$ $(0.1 \%$ to $3.1 \%)$ in participants aged $20-29$ years to $6.0 \%$ $(4.1 \%$ to $8.6 \%)$ in participants $\geq 65$ years of age. ${ }^{23}$

The prevalence of UDM was higher in the present men than women, although the difference was significant only in wt/wt control participants $(6.4 \%$ vs $2.9 \%$, respectively). In regression analyses, male sex was a significant positive predictor for UDM (OR 2.7). In non-Hispanic white NHANES 1999-2002 participants, the standardized prevalence of UDM was significantly higher in men than women $(3.5 \%$ vs $1.9 \%$, respectively). ${ }^{23}$ The prevalence of UDM increased from $0.4 \%$ $(0.1 \%$ to $3.1 \%)$ in men aged $20-29$ years to $6.0 \%(4.1 \%$ to $8.6 \%$ ) in participants $\geq 65$ years of age. ${ }^{23}$

The prevalence of IFG in the present participants without diagnosed diabetes as defined by the American Diabetes Association ${ }^{27}{ }^{28}$ was $21.6 \%$ in p.C282Y homozygotes and $24.2 \%$ in wt/wt controls. The prevalence of IFG was significantly greater in p.C282Y homozygotes and wt/wt control participants $\geq 65$ years of age than participants $<65$ years of age in corresponding genotype groups. There was a significant positive association of age with IFG, although the OR was relatively low. McClain $e t a l^{15}$ evaluated records of homozygous hemochromatosis probands aged $>40$ years who underwent glucose measurements after an overnight fast at the University of Utah in the interval 1975-1998. In 101 Utah probands without diabetes, the prevalence of IFG was $17.8 \% .^{15}$ This prevalence is lower than that in the present p.C282Y homozygotes, possibly due to the more stringent IFG criterion used in the Utah study (fasting glucose $6.00-6.99 \mathrm{mmol} / \mathrm{L}){ }^{15}{ }^{29}$ In non-Hispanic white participants in NHANES 1999-2002, the crude prevalence of IFG defined by the same criterion used here was $27.0 \%$ and ranged from $15.3 \%(11.9 \%$ to $19.3 \%)$ in participants aged $20-29$ years to $40.0 \% \quad(36.2 \%$ to $43.9 \%$ ) in participants $\geq 65$ years of age. ${ }^{23}$

The prevalence of IFG was higher in men than women with UDM in the present p.C282Y homozygotes $(34.1 \%$ vs $16.7 \%$, respectively) and $\mathrm{wt} / \mathrm{wt}$ control

Table 4 Diabetes and impaired fasting glucose in 721 HEIRS Study participants ${ }^{*}$

\begin{tabular}{|c|c|c|c|c|c|}
\hline Classification & $\begin{array}{l}\text { HFE p.C282Y/p.C282Y } \\
(\mathrm{n}=245)\end{array}$ & HFE wt/wt (n=476) & p Value & OR $(95 \% \mathrm{Cl})$ & $\begin{array}{l}\text { Cohen's } \\
\text { h statistic }\end{array}$ \\
\hline $\begin{array}{l}\text { Diagnosed diabetes, } \\
\text { percent }(n)\end{array}$ & 9.0 (5.8 to 13.5$)(22)$ & 5.7 (3.8 to 8.3) (27) & 0.1644 & $\begin{array}{l}1.6(0.9 \text { to } 2.9) \\
p=0.0974\end{array}$ & 0.13 \\
\hline $\begin{array}{l}\text { Undiagnosed diabetes, } \\
\text { percent }(n)\end{array}$ & $3.7(1.8$ to 7.1$)(9)$ & 4.0 (2.5 to 6.3) (19) & 1.0000 & $\begin{array}{l}0.9(0.4 \text { to } 2.1) \\
p=0.8342\end{array}$ & 0.02 \\
\hline $\begin{array}{l}\text { Impaired fasting glucose, } \\
\text { percent }(n)\end{array}$ & 21.6 (16.8 to 27.4$)(53)$ & 24.2 (20.4 to 28.3$)(115)$ & 0.4587 & $\begin{array}{l}0.9(0.6 \text { to } 1.3) \\
p=0.4473\end{array}$ & 0.06 \\
\hline
\end{tabular}

*Proportions were compared using Fisher's exact test (two-tailed). Proportions are displayed as percentage ( $95 \%$ confidence limits; $n$ ). Observations on 721 participants include 672 participants from the present report and 49 HEIRS Study clinical examination participants with diagnosed diabetes described in a previous report (22). Overall prevalences in 721 participants: diagnosed diabetes $6.5 \%(4.9 \%$ to $8.5 \%)$; undiagnosed diabetes $3.9 \%$ (2.6\% to $5.6 \%$ ); and impaired fasting glucose $23.3 \%(20.3 \%$ to $26.6 \%)$.

HEIRS, Hemochromatosis and Iron Overload Screening. 
participants (35.7\% vs $17.1 \%$, respectively). Male sex was a significant positive independent predictor of IFG (OR 2.8). In NHANES 1999-2002 non-Hispanic white participants, the standardized prevalence of IFG was significantly higher in men than women $(33.1 \%$ vs $19.6 \%$, respectively). ${ }^{23}$

HFE genotypes and SF levels were not significantly associated with UDM or IFG in the present study. Likewise, SF and quantities of iron removed by phlebotomy to achieve iron depletion were not significantly associated with type 2 diabetes in p.C282Y homozygotes in a non-screening venue $^{19}$ or in the HEIRS Study. ${ }^{22}$ The prevalence of type 2 diabetes did not differ significantly between p.C282Y homozygotes and corresponding control participants in four population screening studies. ${ }^{30-33}$

The prevalence of diabetes in non-Hispanic white p.C282Y homozygotes diagnosed in screening is similar to that in age-matched and sex-matched HFE wt/wt control subjects, ${ }^{11-13} 24$ although there are no previous substantive reports of UDM and IFG prevalence in p.C282Y homozygotes identified in screening. At the outset of the present clinical examination substudy, we postulated that there were no meaningful differences in the corresponding prevalence proportions of UDM and IFG between HFE genotype subgroups. Previously, when the HEIRS Study was formulated (1998-1999), there were few published estimates of HFE genotype frequencies and no estimates of UDM and IFG in p.C282Y homozygotes identified in screening in the USA. Numbers of HEIRS Study participants and their race/ ethnicity were specified by the National Heart, Lung, and Blood Institute/National Human Genome Research Institute and could not be changed. Thus, ideal numbers of participants invited to the clinical examination could not be ascertained a priori (ie, before screening) and those who would attend the clinical examination could not be predicted. Statistically nonsignificant differences between overall UDM and IFG prevalences (failure to reject the null hypothesis) in the present study are not necessarily equivalent to insufficient power. Post hoc power calculations based on data already gathered and analyzed represent little more than $\mathrm{p}$ value transformations. ${ }^{34-37}$

UDM and IFG increase the risk for the development of type 2 diabetes. ${ }^{38-41}$ Whereas the HEIRS Study is a cross-sectional study, longitudinal studies of other p.C282Y cohorts may provide information about the incidence of UDM and IFG in p.C282Y homozygotes. The prevalences of UDM and IFG in the present non-Hispanic white HEIRS Study participants and in non-Hispanic white participants in the contemporaneous NHANES 1999-2002 ${ }^{23}$ are similar. Regardless, the ages of HEIRS Study participants were $\geq 25$ years $^{24}$ whereas the ages of NHANES 1999-2002 participants were $\geq 20$ years. ${ }^{23}$ There were differences in methods and sites used to recruit and evaluate participants for these respective studies. The present wt/wt control participants were selected because they had initial screening
TS and SF levels between the 25th and 75th centiles of sex-specific distributions. Thus, wt/wt control participants with extremes of TS and SF phenotypes were not represented in our data. Since the present cohort consisted predominantly of middle age and older participants, putative effects associated with younger participants may have been undetected. Hardy-Weinberg analyses of HEIRS Study initial screening data revealed that predicted numbers of p.C282Y homozygotes recruited to the Study were not decreased, ${ }^{25}$ suggesting that there was no selective survivor bias among p.C282Y homozygotes. It is unlikely that having greater numbers of participants in the present study would have revealed meaningful differences not already discovered. The results of our regression analyses indicate that other factors not evaluated in this study also influence the occurrence of UDM and IFG.

\section{CONCLUSIONS}

Prevalences of UDM and IFG were similar in p.C282Y homozygotes and wt/wt control participants in a postpopulation screening examination. Male sex was the strongest predictor of UDM and IFG.

Acknowledgements The authors recognize and appreciate the contributions of all HEIRS Study investigators (online supplementary appendix) and participants. The HEIRS Study (January 2000 to January 2006) was initiated and funded by the National Heart, Lung, and Blood Institute in conjunction with the National Human Genome Research Institute. The study was supported by contracts N01-HC05185 (University of Minnesota); N01-HC05186, N01-CM-07003-74, and the Minority Community Clinical Oncology Program (Howard University); N01-HC05188 (University of Alabama at Birmingham) (RTA, JaCB); N01-C05189 (Kaiser Permanente Center for Health Research); N01-HC05190 (University of California, Irvine); N01-HC05191 (London Health Sciences Centre; PCA); and N01-C05192 (Wake Forest University). Additional support was provided by University of Alabama at Birmingham General Clinical Research Center grant M01-RR00032; and the Southern Iron Disorders Center (JaCB, RTA). The present work was supported in part by Southern Iron Disorders Center.

Contributors JaCB conceived the study, evaluated participants, acquired data, and performed data analyses. JCIB acquired data and performed data analyses. PCA conceived the study, acquired data, and evaluated participants. RTA conceived the study, acquired data, and performed data analyses. All authors drafted the manuscript and approved its final form. JaCB and RTA take responsibility for the contents of the article.

Funding National Heart, Lung, and Blood Institute, National Human Genome Research Institute. Grant number (N01-HC05188 (University of Alabama at Birmingham)).

Competing interests None declared.

Ethics approval IRBs of US NIH/NHLBI/NHGRI and review boards of all HEIRS Study sites.

Provenance and peer review Not commissioned; externally peer reviewed.

Data sharing statement All data from the HEIRS Study are available to qualified investigators via application to the US NHLBI and NHGRI.

Open Access This is an Open Access article distributed in accordance with the Creative Commons Attribution Non Commercial (CC BY-NC 4.0) license, which permits others to distribute, remix, adapt, build upon this work noncommercially, and license their derivative works on different terms, provided the original work is properly cited and the use is non-commercial. See: http:// creativecommons.org/licenses/by-nc/4.0/ 


\section{REFERENCES}

1. Saddi R, Feingold J. Idiopathic haemochromatosis: an autosomal recessive disease. Clin Genet 1974;5:234-41.

2. Edwards CQ, Griffen LM, Goldgar D, et al. Prevalence of hemochromatosis among 11,065 presumably healthy blood donors. N Engl J Med 1988;318:1355-62.

3. Feder JN, Gnirke A, Thomas W, et al. A novel MHC class I-like gene is mutated in patients with hereditary haemochromatosis. Nat Genet 1996;13:399-408.

4. Barton JC, Acton RT, Dawkins FW, et al. Initial screening transferrin saturation values, serum ferritin concentrations, and HFE genotypes in whites and blacks in the Hemochromatosis and Iron Overload Screening Study. Genet Test 2005;9:231-41.

5. Adams PC, Barton JC. How I treat hemochromatosis. Blood 2010;116:317-25.

6. Edwards CQ, Barton JC. Hemochromatosis. In: Greer JP, Arber DA Glader B, List AF, Means RT Jr, Paraskevas F, Rodgers GM, et al. eds. Wintrobe's Clinical Hematology. 13th edn. Philadelphia, Wolters Kluwer/Lippincott Williams \& Wilkins, 2014:662-81.

7. Sheldon JH. Haemochromatosis. Oxford, Oxford University Press, 1935.

8. Finch SC, Finch CA. Idiopathic hemochromatosis, an iron storage disease. A Iron metabolism in hemochromatosis. Medicine (Baltimore) 1955;34:381-430.

9. Hartroft WS. Islet cell pathology in diabetes. Diabetes 1956:5:98-104.

10. Rahier J, Loozen S, Goebbels RM, et al. The haemochromatotic human pancreas: a quantitative immunohistochemical and ultrastructural study. Diabetologia 1987;30:5-12.

11. Åsberg $\mathrm{A}$, Hveem $\mathrm{K}$, Kruger $\mathrm{O}$, et al. Persons with screening-detected haemochromatosis: as healthy as the general population? Scand J Gastroenterol 2002;37:719-24.

12. Beutler E, Felitti VJ, Koziol JA, et al. Penetrance of $845 \mathrm{G}->\mathrm{A}$ (C282Y) HFE hereditary haemochromatosis mutation in the USA. Lancet 2002;359:211-18.

13. Allen KJ, Gurrin LC, Constantine CC, et al. Iron-overload-related disease in HFE hereditary hemochromatosis. N Engl J Med 2008;358:221-30.

14. McLaren GD, McLaren CE, Adams PC, et al. Clinical manifestations of hemochromatosis in HFE C282Y homozygotes identified by screening. Can J Gastroenterol 2008;22:923-30.

15. McClain DA, Abraham D, Rogers J, et al. High prevalence of abnormal glucose homeostasis secondary to decreased insulin secretion in individuals with hereditary haemochromatosis. Diabetologia 2006;49:1661-9.

16. Hramiak IM, Finegood DT, Adams PC. Factors affecting glucose tolerance in hereditary hemochromatosis. Clin Invest Med 1997;20:110-18.

17. Balcerzak SP, Mintz DH, Westerman MP. Diabetes mellitus and idiopathic hemochromatosis. Am J Med Sci 1968;255:53-62.

18. Dymock IW, Cassar J, Pyke DA, et al. Observations on the pathogenesis, complications and treatment of diabetes in 115 cases of haemochromatosis. Am J Med 1972;52:203-10.

19. Barton JC, Barton JC, Acton RT. Diabetes in first-degree family members: a predictor of type 2 diabetes in 159 nonscreening Alabama hemochromatosis probands with HFE C282Y homozygosity. Diabetes Care 2014;37:259-66.

20. Abbas MA, Abraham D, Kushner JP, et al. Anti-obesity and pro-diabetic effects of hemochromatosis. Obesity (Silver Spring) 2014;22:2120-2.

21. Acton RT, Barton JC, Barton JC. Serum ferritin, insulin resistance, and metabolic syndrome: clinical and laboratory associations in 769
non-Hispanic whites without diabetes mellitus in the HEIRS Study. Metab Syndr Relat Disord 2015;13:57-63.

22. Barton JC, Barton JC, Adams PC, et al. Risk factors for insulin resistance, metabolic syndrome, and diabetes in 248 HFE C282Y homozygotes identified by population screening in the HEIRS Study. Metab Syndr Relat Disord 2016;14:94-101.

23. Cowie CC, Rust KF, Byrd-Holt DD, et al. Prevalence of diabetes and impaired fasting glucose in adults in the U.S. population: National Health And Nutrition Examination Survey 1999-2002. Diabetes Care 2006;29:1263-8.

24. McLaren CE, Barton JC, Adams PC, et al. Hemochromatosis and Iron Overload Screening (HEIRS) Study design for an evaluation of 100,000 primary care-based adults. Am J Med Sci 2003;325:53-62.

25. Adams PC, Reboussin DM, Barton JC, et al. Hemochromatosis and iron-overload screening in a racially diverse population. $N$ Engl J Med 2005;352:1769-78.

26. van Straalen JP, Leyte A, Weber JA, et al. Evaluation of the Hitachi 911 for routine urine analysis and for measurement of various special serum analytes. Eur J Clin Chem Clin Biochem 1995;33:315-22.

27. American Diabetes Association. Report of the Expert Committee on the diagnosis and classification of diabetes mellitus. Diabetes Care 1997;20:1183-97.

28. American Diabetes Association. Diagnosis and classification of diabetes mellitus. Diabetes Care 2014;37(Suppl 1):S81-90.

29. Alberti KG, Zimmet PZ. Definition, diagnosis and classification of diabetes mellitus and its complications. Part 1: diagnosis and classification of diabetes mellitus provisional report of a WHO consultation. Diabet Med 1998;15:539-53.

30. Frayling T, Ellard S, Grove J, et al. C282Y mutation in HFE (haemochromatosis) gene and type 2 diabetes. Lancet 1998;351:1933-4.

31. Fernandez-Real JM, Vendrell J, Baiget M, et al. C282Y and H63D mutations of the hemochromatosis candidate gene in type 2 diabetes. Diabetes Care 1999;22:525-6.

32. Sampson MJ, Williams T, Heyburn PJ, et al. Prevalence of HFE (hemochromatosis gene) mutations in unselected Male patients with type 2 diabetes. J Lab Clin Med 2000;135:170-3.

33. Moczulski DK, Grzeszczak W, Gawlik B. Role of hemochromatosis C282Y and H63D mutations in HFE gene in development of type 2 diabetes and diabetic nephropathy. Diabetes Care $2001 ; 24: 1187-91$

34. Thomas L. Retrospective power analysis. Conservation Biology 1997;11:276-80.

35. Hoenig JM, Heisey DM. The abuse of power: the pervasive fallacy of power calculations for data analysis. Am Stat 2001;55:19-24.

36. Levine M, Ensom MH. Post hoc power analysis: an idea whose time has passed? Pharmacotherapy 2001;21:405-9.

37. Sullivan GM, Feinn R. Using effect size-or why the p value is not enough. J Grad Med Educ 2012;4:279-82.

38. Nichols GA, Hillier TA, Brown JB. Progression from newly acquired impaired fasting glucose to type 2 diabetes. Diabetes Care 2007;30:228-33.

39. Karve A, Hayward RA. Prevalence, diagnosis, and treatment of impaired fasting glucose and impaired glucose tolerance in nondiabetic U.S. adults. Diabetes Care 2010;33:2355-9.

40. Morris $\mathrm{DH}$, Khunti K, Achana F, et al. Progression rates from $\mathrm{HbA1c}$ 6.0-6.4\% and other prediabetes definitions to type 2 diabetes: a meta-analysis. Diabetologia 2013;56:1489-93.

41. Selph S, Dana T, Blazina I, et al. Screening for type 2 diabetes mellitus: a systematic review for the U.S. Preventive Services Task Force. Ann Intern Med 2015;162:765-76. 